\title{
The Impact of Blue Inorganic Pigments on the Microwave Electrical Properties of Polymer Composites
}

\author{
L. C. Costa $^{1}$ and F. Henry ${ }^{2}$ \\ ${ }^{1}$ I3N and Physics Department, University of Aveiro, 3810-193 Aveiro, Portugal \\ ${ }^{2}$ LRP, CNRS, 2 rue Henry Dunand, 94320 Thiais, France \\ Correspondence should be addressed to L.C. Costa, kady@ua.pt
}

Received 5 October 2011; Accepted 16 January 2012

Academic Editor: Paolo Colantonio

Copyright (C) 2012 L. C. Costa and F. Henry. This is an open access article distributed under the Creative Commons Attribution License, which permits unrestricted use, distribution, and reproduction in any medium, provided the original work is properly cited.

\begin{abstract}
We present the results of the measurement of complex dielectric permittivity, in the microwave frequency region, on glass reinforced polybutylene terephthalate (PBT) with blue inorganic pigments. The cavity resonant method had been used in order to measure the shift in the resonant frequency of the cavity, caused by the insertion of a sample, which can be related to the real part of the complex permittivity. Also, the quality factor of the cavity decreases with the insertion of a sample. The changes in the inverse of this quality factor give the imaginary part. In order to predict the dielectric behavior of this composite, we had developed a program of numerical simulation to calculate the complex permittivity of the inclusion. By using some of dielectric mixture laws (Maxwell-Wagner-Sillars, Hanai, Looyenga, inverse and direct Wiener, and Bruggemann), we can predict the dielectric behavior of the composite in a large range of volume fraction of inclusions.
\end{abstract}

\section{Introduction}

Ultramarine blue is a nonhazardous pigment with a lot of variety of industrial applications. Its manufacturing process and possibility for close control over its physical, chemical, and particular color characteristics enable the production of several types of this blue pigment, which are readily accepted by polymers, printing ink, paint, cosmetic, and many other industries, due to advantages over other organic pigments.

The electrical conductivity of an insulating polymer, such as polybutylene terephtalate (PBT), can be altered by adding different conducting particles, like carbon [1-3], iron [4], nickel [5], or another conducting polymer [6-8]. The conductivity of the composite material can thus be controlled by properly choosing the components, their shape, and their relative volume fractions.

Small quantities of conducting particles can increase the dielectric constant, without exceeding the critical concentration of percolation [9], that is, avoiding high conductivity. If electrical losses become high, heating of the plastic will occur, resulting in melting or even carbonization.
For high frequencies, as in the microwave range, interfacial polarization mechanisms are not present, because such polarization occurs at frequencies lower than the times scales typical of dipolar polarizations [10].

In order to predict the electrical properties of the composite, different mixture laws can be used. Numerical simulations of mixtures can lead not only to a better understanding of the physics of dielectrics but also to improvement of designing of tailored materials without going to expensive attempts.

Numerous authors have studied the dielectric behavior of nonhomogeneous materials [11-13], and several theories can be applied, depending on the difference of the electrical properties of the host and the inclusion materials $[14,15]$.

\section{Experimental}

The blue inorganic pigment, with chemical formula $\mathrm{Na}_{6} \mathrm{Al}_{6} \mathrm{Si}_{6} \mathrm{O}_{24} \mathrm{~S}_{4}$, in the form of powder with mean particle size $3.8 \mu \mathrm{m}$, was obtained from Kremer Pigments Inc., USA. Glass fibers of 10 to $20 \mu \mathrm{m}$ reinforced the polybutylene terephthalate matrix, which was purchased from DuPont, 
USA. The powder samples were pressed at room temperature and $4 \mathrm{MPa}$ to obtain cylinders of length $10 \mathrm{~mm}$ and diameter $4 \mathrm{~mm}$.

The resonant cavity method was used to calculate the complex permittivity $[16,17], \varepsilon^{*}=\varepsilon^{\prime}-i \varepsilon^{\prime \prime}$. Two different rectangular cavities were used, operating in $\mathrm{TE}_{3,5,5}$ and $\mathrm{TE}_{0,1,11}$ modes, and resonant frequencies of 2.7 and $5 \mathrm{GHz}$, respectively. In this technique, we measured the shift in the cavity resonant frequency, $\Delta f$, caused by the insertion of the sample inside the cavity, which can be related to the real part of the complex permittivity, $\varepsilon^{\prime}$, and the change in the inverse of the quality factor of the cavity, $\Delta(1 / Q)$, which is related with the imaginary part, $\varepsilon^{\prime \prime}$. The relations are simple when we consider only the first-order perturbation in the electric field caused by the sample [18],

$$
\frac{\Delta f}{f_{0}}+i \Delta\left(\frac{1}{2 Q}\right)=\varepsilon_{0}\left(\varepsilon^{*}-1\right) \frac{\int_{V_{s}} E_{i}{ }^{*} E_{0} d V}{\int_{V} \varepsilon_{0} E_{0}^{2} d V},
$$

where $f_{0}$ is the resonance frequency of the cavity, $\varepsilon^{*}$ the complex permittivity of the material, and $E_{i}$ and $E_{0}$ the electric fields inside and outside the material. The integration is made in the volume of the sample, $V_{s}$, and in the volume of the cavity, $V$.

Splitting the real and imaginary parts, we can obtain the expressions for $\varepsilon^{\prime}$ and $\varepsilon^{\prime \prime}$,

$$
\begin{aligned}
\varepsilon^{\prime} & =K \frac{\Delta f}{f_{0}} \frac{V}{V_{s}}+1, \\
\varepsilon^{\prime \prime} & =\frac{K}{2} \Delta\left(\frac{1}{Q}\right) \frac{V}{V_{s}},
\end{aligned}
$$

where $K$ is a constant related to the depolarization factor, which depends upon the geometric parameters. In fact, this factor introduces the effect of the shape and dimensions of the sample in the electromagnetic field perturbation. Maxwell equations and the boundary conditions were used to deduce the previous equations [19], taking into account the depolarization field, which appears outside the dielectric. To calculate the factor $K$, we used a sample of known dielectric constant of polytetrafluorethylene (PTFE) $\left(\varepsilon^{\prime}=2.1\right.$ at microwave frequencies), with the same size and shape of the studied samples.

In order to couple the microwave to the cavity, we used the quarter-wavelength flange joints and small circular irises (10 $\mathrm{mm}$ in diameter).

For the measurements, we used an HP 8753D Network Analyzer with the excitation power of $1 \mathrm{~mW}$. All the measurements were carried out at constant temperature. The measured transmissions are the $S_{12}$ parameters that quantify how the microwave energy propagates through a multiport network. The subscripts " 12 " refers to the ratio of signal from port 1 (input) to port 2 (output).

The samples, ultramarine blue pigments, in concentrations up to $4 \%$ in a glass reinforced polybutylene terephtalate matrix, were introduced into the cavity through circular holes milled in the centre of the cavity. There, the electric field is in its strongest value and the insertion of the samples causes the maximal frequency shift. That is, a coupling between the samples and the electric field is produced

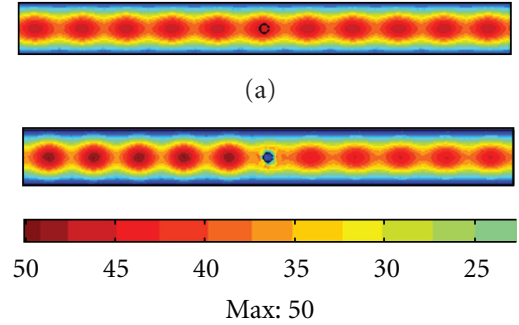

(b)

FIGURE 1: Calculated electric field in the $5 \mathrm{GHz}$ cavity, without (a) and with a sample in the center (b). The vertical bar shows that the highest electric field is about $50 \mathrm{kVm}^{-1}$.

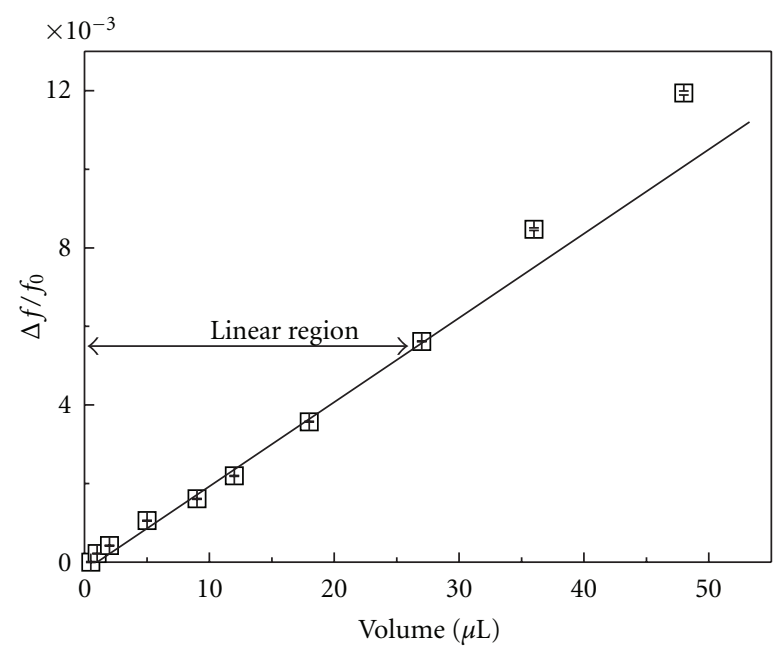

Figure 2: $\Delta f / f_{0}$ versus volume of water. $f_{0}$ is the resonance frequency of the cavity and $\Delta f$ the variation of resonant frequency by the insertion of the samples.

in this region [19], resulting in a small perturbation of that field. The interest lies in the cases in which the samples are homogeneous and thier volumes are very small compared with the volume of the cavity. Figure 1 shows the electric field distribution in the $5 \mathrm{GHz}$ cavity, without and with a sample, in a simulation made using the COMSOL software. The changes in the electric field in the center of the cavity, due to by the insertion of a sample in this region, are clearly visible, when comparing Figures 1(a) and 1(b).

\section{Results}

To study the linearity of the cavity, and consequently to infer the possibility to use the small perturbation theory [19], we carried out measurements using glass microtubes filled with distilled water. In Figures 2 and 3 we present, as an example, $\Delta f / f_{0}$ and $\Delta(1 / Q)$ for the different volume of distilled water ( $5 \mathrm{GHz}$ resonant cavity), and the linear fit parameters that confirm the possibility of using (2).

The shift in the resonant frequency of the cavity remains in the linear regime for measurements up to $27 \mu \mathrm{L}$ of water, which corresponds to $\Delta f / f_{0}$ of $0.56 \%$. The linearity in the 


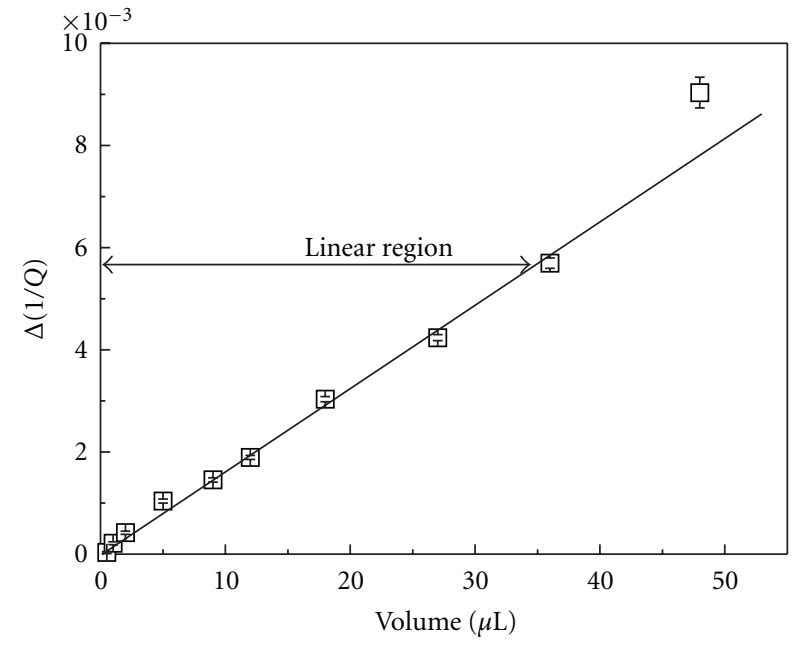

Figure 3: $\Delta(1 / Q)$ versus volume of water. $Q$ is quality factor of the cavity, which is degraded by the insertion of the samples.

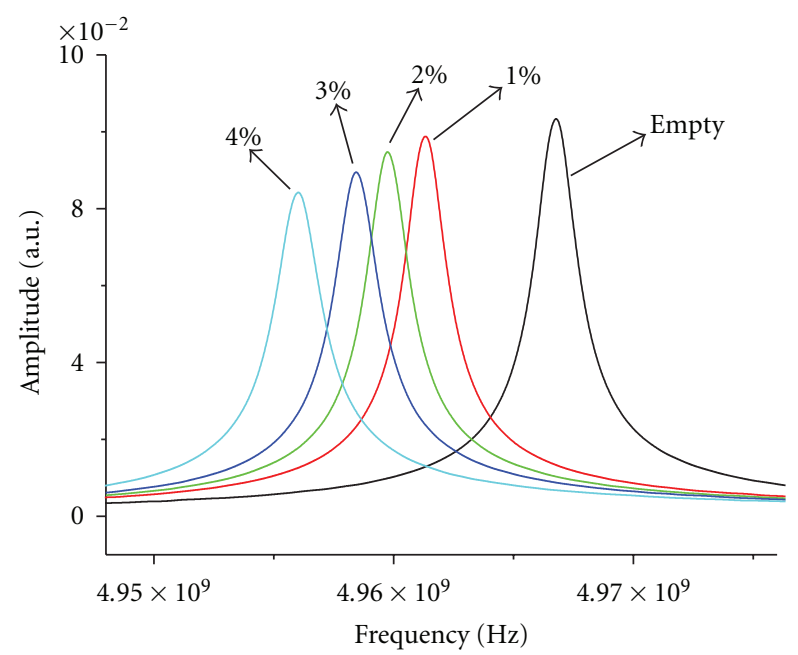

FIGURE 4: Transmission for the empty and loaded cavity with samples of different concentrations of blue pigments.

inverse of the quality factor is observed up $36 \mu \mathrm{L}$ that is up to $5.7 \times 10^{-3}$. The conjugation of both conditions permits us to conclude that the cavity can be used up to variations of $\Delta f / f_{0}$ of $0.56 \%$ and $\Delta(1 / Q)$ of about $4.2 \times 10^{-3}$. If the measurements overcome this limit, the sample volume should be reduced until the results falls into the linear regime [20].

In Figure 4 we show the transmissions of the $5 \mathrm{GHz}$ cavity, for the empty and loaded cavity with samples of different concentrations of blue pigments. The most perturbating sample was referred to that with highest concentration of blue inorganic pigments, corresponding to the highest complex permittivity. This means that the real and imaginary parts of the complex permittivity of the filler has considerable values.

\section{Discussion}

A very common mixture law was proposed by Hanai [21]. If the filler particles, with a complex permittivity $\varepsilon_{f}^{*}(\omega)$ and volume fraction $\varphi_{f}$ are dispersed in a matrix material with complex permittivity $\varepsilon_{m}^{*}(\omega)$ and volume fraction $\varphi_{m}$, then, according to this theory,

$$
\frac{\varepsilon_{f}^{*}-\varepsilon^{*}}{\varepsilon_{f}^{*}-\varepsilon_{m}^{*}}\left(\frac{\varepsilon_{m}^{*}}{\varepsilon^{*}}\right)^{1 / 3}=1-\varphi_{f} .
$$

Another theory, developed by Maxwell-Wagner-Sillars predicts the polarization process due to differences in conductivity and permittivity of the constituents [22]. The complex permittivity of the mixture can be calculated from

$$
\varepsilon^{*}=\varepsilon_{m}^{*} \frac{\left(n \varepsilon_{f}^{*} *+(1-n) \varepsilon_{m}^{*}\right)+(1-n)\left(\varepsilon_{f}^{*}-\varepsilon_{m}^{*}\right) \varphi_{f}}{\left(n \varepsilon_{f}^{*}+(1-n) \varepsilon_{m}^{*}\right)-n\left(\varepsilon_{f}^{*}-\varepsilon_{m}^{*}\right) \varphi_{f}},
$$

in which $n$, with $0 \leq n \leq 1$, is the shape factor of the dispersed particles in the direction of the electrical field lines. For spherical particles, $n=1 / 3$ [23].

The Bruggeman effective medium theory has the virtue of simplicity. It can be used with the assumption that the inclusions do not interact each other and that are randomly distributed in the matrix [24]. In the most commonly used form, that is, the implicit form,

$$
\varphi_{f} \frac{\varepsilon_{f}^{*}-\varepsilon^{*}}{\varepsilon_{f}^{*}+A \varepsilon^{*}}+\left(1-\varphi_{f}\right) \frac{\varepsilon_{m}^{*}-\varepsilon^{*}}{\varepsilon_{m}^{*}+A \varepsilon^{*}}=0,
$$

where, for spherical particles embedded in a host matrix, $A=$ 2. This law predicts a percolation threshold of conduction for $\varphi_{f}=1 /(A+3)$.

Looyenga introduced a new mixture formula [25], usually known as generalized Looyenga law,

$$
\varepsilon^{*}(\omega)^{1 / t}=\varphi_{f} \varepsilon_{f}^{*}(\omega)^{1 / t}+\varphi_{m} \varepsilon_{m}^{*}(\omega)^{1 / t},
$$

where for spherical inclusions $t=3$. For $t= \pm 1$, we arrive to the Wiener laws (direct and inverse) [15].

Table 1 summarizes the obtained results for the fit of the data with the four previous laws. The values for the real and imaginary parts of the complex permittivity of the charge are indicative that generalized Looyenga model is more accurate, which is confirmed by the obtained values of the standard deviation $\chi^{2}$.

Figure 5 shows the measured real and imaginary parts of the complex permittivity for different concentrations of the blue inorganic pigment, at $2.7 \mathrm{GHz}$, and the fit with the different mixture laws, confirming the good accuracy in the fit using generalized Looeynga. Similar results, from the point of view of quality of the fit, are obtained for the $5 \mathrm{GHz}$ measurements frequency.

For these small quantities of blue inorganic particles, the percolation threshold is not observed and the composite is maintained as an insulator.

Finally, the small polarity present in PBT explains the measured complex permittivity. The obtained values make 
TABLE 1: Calculated values for $\varepsilon_{f}^{\prime}$ and $\varepsilon_{f}^{\prime \prime}$ of the filler, and standard deviation $\chi^{2}$, using the inversion of Wiener, Hanai, Looyenga, MaxwellWagner-Sillars, and Bruggeman laws. $A$ is a parameter of Bruggeman and $t$ the exponent of generalized Looyenga laws.

\begin{tabular}{|c|c|c|c|c|c|c|c|}
\hline & Parameters & Direct Wiener & Invere Wienr & Hanai & Maxwell-Wagner-Sillars & Generalized Looyenga & Bruggeman \\
\hline \multirow{5}{*}{$2.7 \mathrm{GHz}$} & $\varepsilon_{f}^{\prime}$ & 23.63 & $6.13 \mathrm{E} 5$ & 2.75 & $9.87 \mathrm{E} 5$ & 108.3 & 52.1 \\
\hline & $\varepsilon_{f}^{\prime \prime}$ & 3.72 & - & 0.42 & $1.24 \mathrm{E} 5$ & 39.58 & 19.18 \\
\hline & $t$ & - & - & - & - & 3.50 & - \\
\hline & $A$ & - & - & - & - & - & 7.97 \\
\hline & $\chi^{2}$ & 0.0218 & 0.2455 & 0.2901 & 0.1627 & 0.0226 & 0.0248 \\
\hline \multirow{5}{*}{$5 \mathrm{GHz}$} & $\varepsilon_{f}^{\prime}$ & 22.36 & 7.3E5 & $1.1 \mathrm{E} 5$ & 1.0 & 106.9 & 1.0 \\
\hline & $\varepsilon_{f}^{\prime \prime}$ & 0.214 & $8.0 \mathrm{E} 5$ & 354.6 & 355.0 & 102.3 & 155.1 \\
\hline & $t$ & - & - & - & - & 3.94 & - \\
\hline & $A$ & - & - & - & - & - & 4.86 \\
\hline & $\chi^{2}$ & 0.0446 & 0.2289 & 0.1381 & 0.1402 & 0.0415 & 0.0780 \\
\hline
\end{tabular}
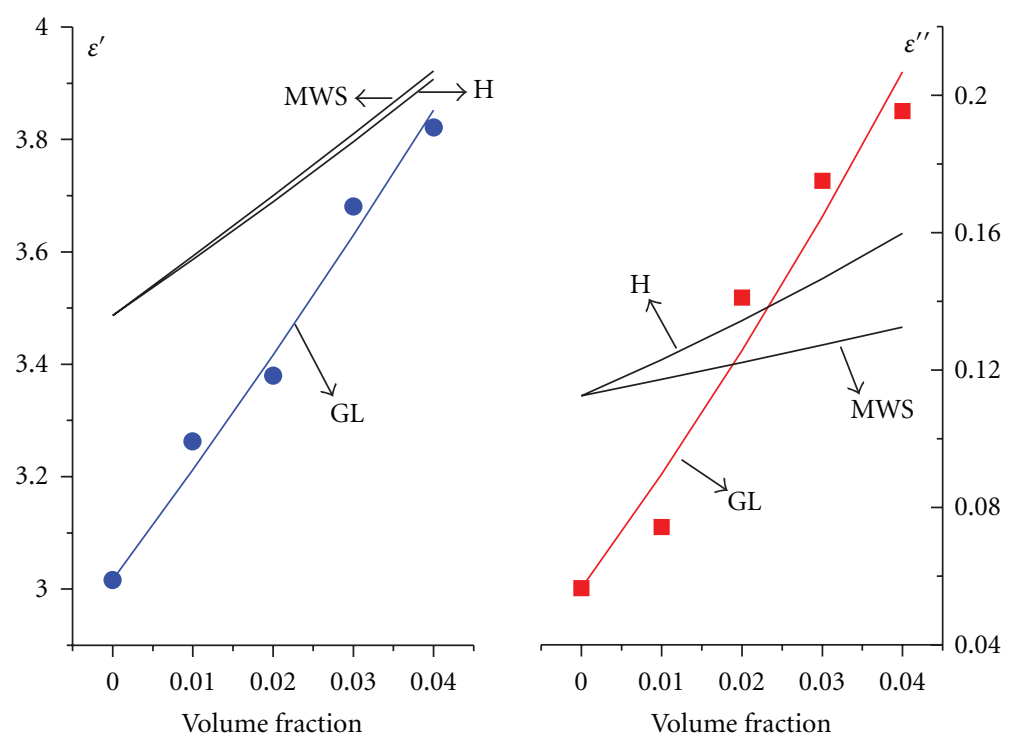

Figure 5: Measured real and imaginary parts of the complex permittivity for different concentrations of the blue inorganic pigment, at 2.7 GHz, and the fit with the generalized Looyenga (GL), Hanai (H), and Maxwell-Wagner-Sillars (MWS) mixture laws.

this material a good choice for applications in telecommunications products. The higher $\varepsilon^{\prime}$ permits to reduce the dimensions of the rods in dielectric antennas. In espite of the higher $\varepsilon^{\prime \prime}$, the values are still quite low, avoiding the heating of the material.

\section{Conclusions}

The cavity perturbation method presents a good accuracy to evaluate the dielectric permittivity of low loss materials.

The dielectric function of two phase materials can be accurately deduced using the generalized Looyenga model, in particular when the inclusion is conductive, and in low filler volume fractions. In this case, the percolation critical concentration is not reached.

With this law, we can choose the adequate doping concentrations, and then to control the electrical properties, in order to obtain the desired behavior, for a particular application.

\section{References}

[1] C. Brosseau, F. Boulic, P. Queffelec et al., "Dielectric and microstructure properties of polymer carbon black composites," Journal of Applied Physics, vol. 81, no. 2, pp. 882-891, 1997.

[2] I. Krupa and I. Chodak, "Physical properties of thermoplastic/graphite composites," European Polymer Journal, vol. 37, no. 11, pp. 2159-2168, 2001.

[3] G. Chen, D. Wu, W. Weng, H. Bin, and W. Yan, "Preparation of polystyrene-graphite conducting nanocompositesvia intercalation polymerization," Polymer International, vol. 50, no. 9, pp. 980-985, 2001.

[4] L. Costa, M. Valente, F. Henry, and L. Ramanitra, "Electrical properties of the polymer composite polystyrene/iron particles," Journal de Chimie Physique et de Physico-Chimie Biologique, vol. 95, no. 6, pp. 1453-1456, 1998.

[5] A. A. Khoroshilov, I. P. Koroleva, and Y. Y. Volodin, "Nickel/ polystyrene composite electrode materials," Russian Journal of Applied Chemistry, vol. 73, no. 11, pp. 1918-1921, 2000. 
[6] L. C. Costa, F. Henry, and A. André, "Propriétés électrique et mécanique de matériaux nanocomposites à polymère conducteur," in Proceedings of the Journées Polymères Conducteurs, Collonges la Rouge, France, 1996.

[7] G. Street, Handbook of Conducting Polymers, Dekker, New York, NY, USA, 1986.

[8] Y. Roichman, M. S. Silverstein, A. Siegmann, and M. Narkis, "Percolation of electrical conductivity in solution-cast blends containing polyaniline," Journal of Macromolecular Science, vol. B38, no. 1-2, pp. 145-161, 1999.

[9] M. A. Valente, L. C. Costa, S. K. Mendiratta, F. Henry, and L. Ramanitra, "Structural and electrical properties of polystyrene-carbon composites," Solid State Communications, vol. 112, no. 2, pp. 67-72, 1999.

[10] A. Schonhals and F. Kremer, "Analysis of dielectric spectra," in Broadband Dielectric Spectroscopy, F. Kremer and A. Schonhals, Eds., Springer, Berlin, Germany, 2003.

[11] G. Bánhegyi, "Comparison of electrical mixture rules for composites," Colloid \& Polymer Science, vol. 264, no. 12, pp. 1030-1050, 1986.

[12] D. K. Hale, "The physical properties of composite materials," Journal of Materials Science, vol. 11, no. 11, pp. 2105-2141, 1976.

[13] F. Henry, F. Cansell, J. L. Guillaume, and C. Pichot, "Study of the polymer latex coalescence by dielectric measurements at microwave frequency-feasibility of the method," Colloid \& Polymer Science, vol. 267, no. 2, pp. 167-178, 1989.

[14] Y. Roichman, M. S. Silverstein, A. Siegmann, and M. Narkis, "Percolation of electrical conductivity in solution-cast blends containing polyaniline," Journal of Macromolecular Science, vol. B38, no. 1-2, pp. 145-161, 1999.

[15] F. Henry and L. C. Costa, "Percolation and order-disorder transition," Physica B, vol. 387, no. 1-2, pp. 250-258, 2007.

[16] A. Rejou-Michel, F. Henry, M. De Villardi, and M. Delmotte, "Protein and ion hydration variation in fixed aqueous solutions: measurement by dielectric decrement," Physics in Medicine and Biology, vol. 30, no. 8, pp. 831-837, 1985.

[17] C. P. L. Rubinger and L. C. Costa, "Building a resonant cavity for the measurement of microwave dielectric permittivity of high loss materials," Microwave and Optical Technology Letters, vol. 49, no. 7, pp. 1687-1690, 2007.

[18] D. S. McLachlan, A. Priou, I. Chennerie, E. Isaac, and F. Henry, "Modeling the permittivity of composite materials with a general effective medium equation," Journal of Electromagnetic Waves and Applications, vol. 6, no. 9, pp. 1099-1131, 1992.

[19] D. X. Gouveia, L. C. Costa, and M. A. Valente, "Resonant cavity for the measurement of microwave magnetic permeability using the small perturbation theory," Microwave and Optical Technology Letters, vol. 50, no. 2, pp. 399-402, 2008.

[20] L. C. Costa, S. Devesa, P. André, and F. Henry, "Microwave dielectric properties of polybutylene terephtalate (PBT) with carbon black particles," Microwave and Optical Technology Letters, vol. 46, no. 1, pp. 61-63, 2005.

[21] T. Hanai, "Theory of the dielectric dispersion due to interfacial polarisation and its application to emulsions," KolloidZeitschrift, vol. 171, pp. 23-31, 1960.

[22] R. W. Sillars, "The behaviour of polar molecules in solid paraffin wax. Ibid," Proceedings of Real Society of London, vol. 169, p. 66, 1939.

[23] V. Beek, "Dielectric behaviour of heterogeneous systems," in Progress in Dielectrics, J. B. Birks, Ed., pp. 69-87, Heywood Books, London, UK, 1967.
[24] C. Brosseau, P. Quéffélec, and P. Talbot, "Microwave characterization of filled polymers," Journal of Applied Physics, vol. 89, no. 8, pp. 4532-4540, 2001.

[25] H. Looyenga, "Dielectric constants of heterogeneous mixtures," Physica, vol. 31, no. 3, pp. 401-406, 1965. 

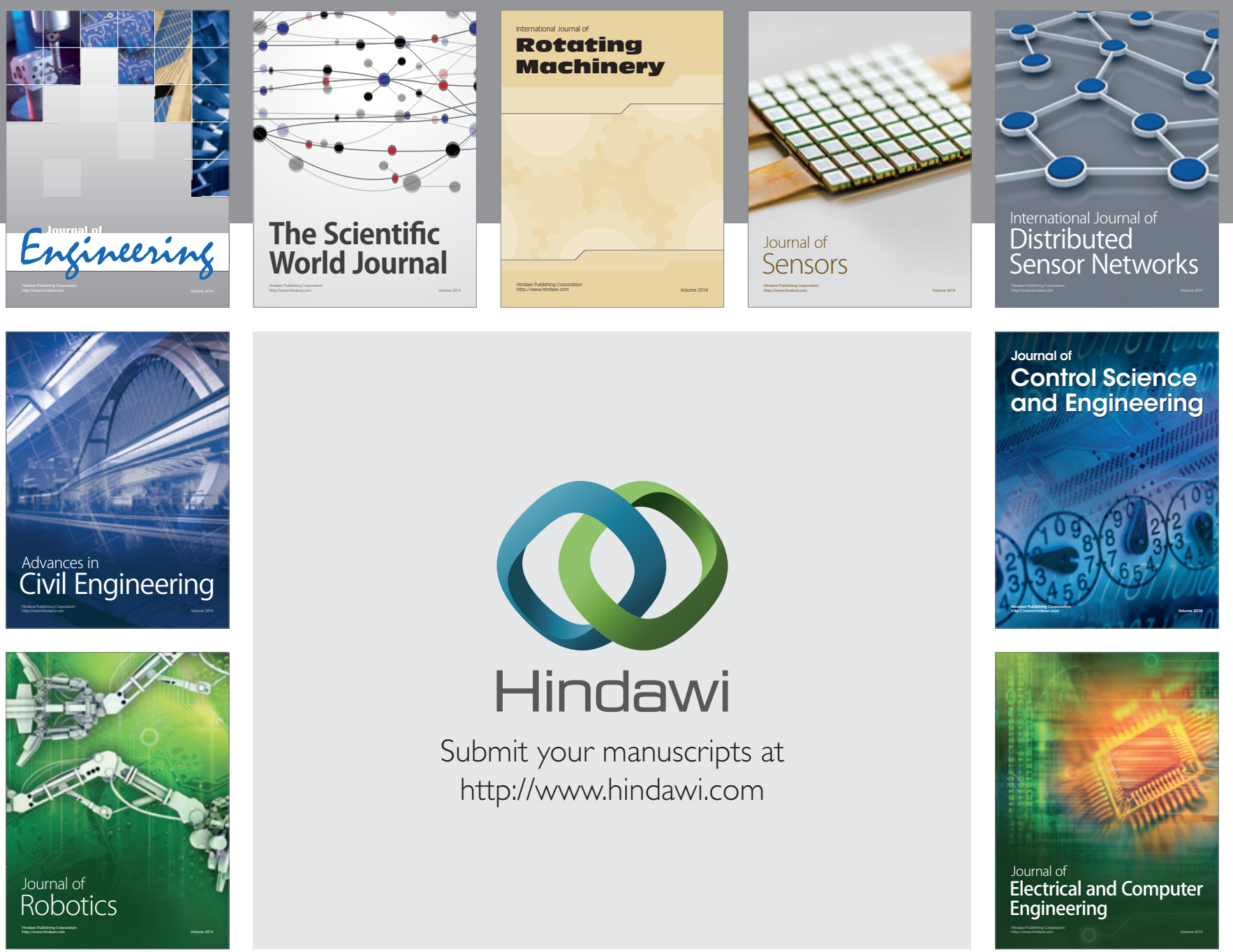

Submit your manuscripts at

http://www.hindawi.com
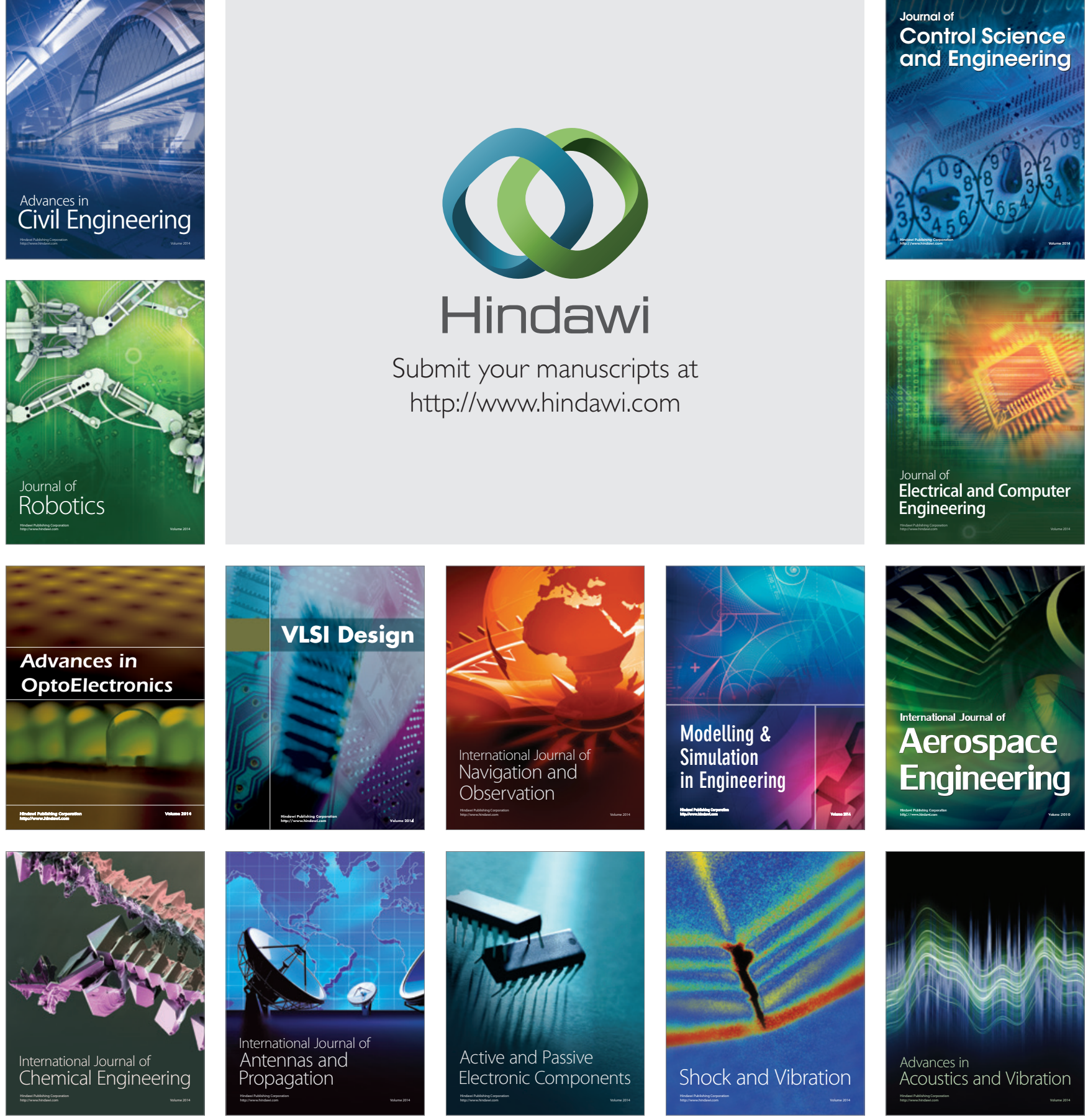